\title{
First fish fauna assessment in the Fernando de Noronha Archipelago with BRUVS: Species catalog with underwater imagery
}

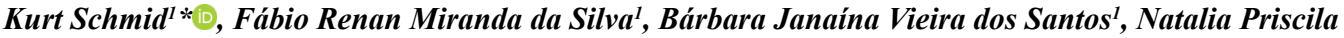 \\ Alves Bezerra ${ }^{2}$, Ricardo Clapis Garla ${ }^{3} \mathbb{D}$ \& Tommaso Giarrizzo ${ }^{1,4}$ [C] \\ ${ }^{1}$ Universidade Federal do Pará, Núcleo de Ecologia Aquática e Pesca da Amazônia, Grupo de Ecologia Aquática, \\ Av. Perimetral, Terra Firme, 66075-110, Belém, PA, Brasil. \\ ${ }^{2}$ Universidade Federal Rural de Pernambuco, Departamento de Pesca e Aquicultura, Rua Dom Manuel de Medeiros, \\ Dois Irmãos, 52171-900, Recife, PE, Brasil. \\ ${ }^{3}$ Universidade Federal do Rio Grande do Norte, Departamento de Botânica e Zoologia, 59078-970, Natal, RN, Brasil. \\ ${ }^{4}$ Instituto Bicho D'água Conservação Socioambiental, Belém, PA, Brasil. \\ *Corresponding author:KurtSchmid, e-mail:kurtschmid@hotmail.com
}

SCHMID, K., SILVA, F.R.M., SANTOS, B.J.V., BEZERRA, N.P.A., GARLA, R.C., GIARRIZZO, T. First fish fauna assessment in the Fernando de Noronha Archipelago with BRUVS: Species catalog with underwater imagery. Biota Neotropica 20(4): e20201014. https://doi.org/10.1590/1676-0611-BN-2020-1014

\begin{abstract}
Fish fauna assessments with traditional catch methods are undesired in marine reserves. Underwater visual census on the other hand is biased due to fish-diver interactions, such as shyness or avoidance behavior of large-bodied target species. This study presents the first marine ichthyofauna inventory of the Fernando de Noronha Archipelago, off northeastern Brazil, sampled with non-destructive, independent Baited Remote Underwater Video Systems (BRUVS). High fish species richness, totaling 66 species from 28 families and 10 orders, including five elasmobranch species and other large predatory fish, such as barracudas, groupers and snappers, were recorded from 81 underwater video samples. Additionally, all of the 4,398 individuals sighted were associated to up to four different benthic habitat types. A catalog of underwater fish images and a detailed species list with additional information, such as conservation status, are provided.
\end{abstract}

Keywords: Ichthyofauna; Biodiversity; Sampling methods; Conservation.

\section{Primeira avaliação da fauna de peixes no Arquipélago de Fernando de Noronha com BRUVS: Catálogo de espécies com imagens subaquáticas}

Resumo: Inventários de ictiofauna com métodos tradicionais de captura são indesejáveis em reservas marinhas. O censo visual subaquático, porém, sofre o viés das interações peixe-mergulhador, tais como comportamentos de timidez ou fuga exercido pelas grandes espécies-alvo de pressões pesqueiras, incluindo, a caça submarina. Esse estudo representa o primeiro inventário da ictiofauna marinha do Arquipélago de Fernando de Noronha, costa nordeste do Brasil, utilizando sistemas não-destrutivos independentes de vídeos subaquáticos com iscas (BRUVS). Uma elevada riqueza de espécies de peixes, com 66 espécies de 28 famílias e 10 ordens, incluindo cinco espécies de elasmobrânquios e outros grandes peixes predadores como barracudas, garoupas e dentões, foi registrada em 81 amostras de vídeos subaquáticos. Adicionalmente, todos os 4.398 indivíduos avistados foram associados a até quatro diferentes tipos de habitats bentônicos. Um catálogo de imagens subaquáticas dos peixes e uma lista de espécies detalhada com informações adicionais, tais como o status de conservação, são fornecidos.

Palavras-chave: Ictiofauna; Biodiversidade; Métodos de amostragem; Conservação. 


\section{Introduction}

The Fernando de Noronha Archipelago (FN) located in the Equatorial Western Atlantic Ocean, off Brazil's northeastern coast, contains a Marine National Park (PARNAMAR) to protect its important marine and terrestrial biodiversity (Soto, 2001; Floeter et al., 2001; IBAMA, 1990; ICMBio, 2017). The reef fish fauna of FN presents a considerable level of endemism (6.3\%) (Floeter \& Gasparini, 2000; Floeter et al., 2001). Recently, Pinheiro et al. (2018) found that Fernando de Noronha Archipelago may share endemic species with insular regions as far away as Trindade, located $1.860 \mathrm{~km}$ south of FN, highlighting the importance of the island in harboring a unique and rich marine biodiversity.

One of the most complete ichthyofauna inventories of the Fernando de Noronha Archipelago and its surroundings was developed based on existing literature and three scientific expeditions (ARFENOR I, II and III), where a combination of visual census with scuba, snorkeling, and fisheries inventory was employed (Soto, 2001). More recent inventories were carried out using visual census with scuba gear to assess the reef fishes of FN (Krajewski \& Floeter, 2011; Medeiros et al., 2011), or snorkeling, focusing on the fish fauna of shallow reef areas (Ilarri et al., 2017). Additionally, the intertidal fishes of FN were studied by Andrades et al. (2018) in an assemblage structure approach.

Stressful sampling techniques, inducing the capture and sacrifice of high numbers of specimens is undesirable in marine protected areas, specifically in aquatic ecosystems harboring threatened and endemic species, such as FN (Floeter \& Gasparini, 2000; Floeter et al., 2001; Andrades et al., 2018). In regard to traditional underwater visual census, a widespread technique to study reef fishes, fish-scuba diver interactions are a common methodological bias (Kulbicki, 1998; Harvey et al., 2002). This is specifically problematic when studying large bodied species, such as groupers, snappers and parrotfishes (Gotanda et al., 2009; Januchowski-Hartley et al., 2011), commonly targeted along the tropical coast of Brazil (Nunes et al., 2012, 2016).

Sampling the highest possible number of species inhabiting a certain study area is paramount in a fish fauna inventory. As exposed above with studies in FN, focusing on distinct components of the local fish fauna, this may require a combination of different sampling methods, in order to achieve this. In this sense, all possible method-related limitations should be avoided, by also employing alternative methods (Willis \& Babcock, 2000; Cappo et al., 2003). Baited remote baited underwater video systems (BRUVS) can offer such an alternative, non-destructive, non-lethal and efficient fish fauna sampling technique, that additionally bypasses the limitations and risks inherent to scuba diving (Willis \& Babcock, 2000; Cappo et al., 2003; Lindfield et al., 2014). In this context, the present study provides an inventory of the ichthyofauna of the Fernando de Noronha Archipelago, using data from BRUVS sampling in different benthic habitats, and supplementary species images.

\section{Material and Methods}

\section{Study Area}

This study was conducted in the Fernando de Noronha Archipelago (FN), an isolated group of volcanic islands, located in the western tropical Atlantic $345 \mathrm{~km}$ off the north-eastern coast of Brazil $\left(03^{\circ} 52^{\prime} \mathrm{S} ; 32^{\circ} 25^{\prime} \mathrm{W}\right)$ (Figure 1). About $70 \%$ of the main island and the waters spanning its coastline to the $50 \mathrm{~m}$ isobath constitute as a notake zone (Figure 1), declared a National Marine Park (corresponding to IUCN Category II) in 1988 (IBAMA, 1990, Maida, Ferreira, 1997, Dudley, 2008). The remaining portion of FN is an Environmental Protection Area (EPA) designated for sustainable use (corresponding to IUCN Category V) established in 1986 (IBAMA, 1990, Maida, Ferreira, 1997, Dudley, 2008). The archipelago is under the influence of the South Equatorial Current, with annual averages of water temperature and salinity of $26^{\circ} \mathrm{C}$ and $36 \%$, respectively. The archipelago has two main underwater landscapes: the windward side, characterized by extensive algal-vermetid reef barriers along rocky shorelines, and the leeward side, mainly composed of descending slopes along a rocky shoreline with large scattered boulders and sparse and small reefs (Maida, Ferreira, 1997).

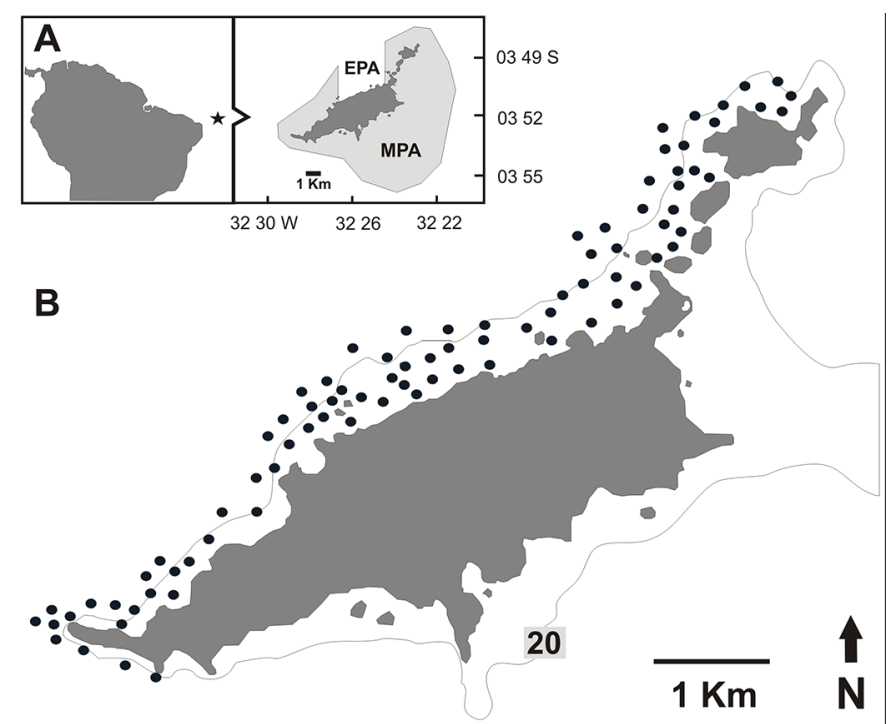

Figure 1. Study area map at the Fernando de Noronha Archipelago with sampling locations (black dots), $20 \mathrm{~m}$ depth isobaths (20) and delimitation of the areas inside (MPA) and outside (EPA) of the Marine National Park (PARNAMAR) in the right small window

\section{BRUVS sampling}

In August 2017, 81 single BRUVS (one camera) deployments were realized in order to sample the FN ichthyofauna. Deployments were made randomly over a variety of benthic habitats, in depths ranging from approximately 5 to $35 \mathrm{~m}$. BRUVS sampling was conducted at least 1 hour after sunrise and 1 hour before sunset due to visibility conditions and to remove the effect of crepuscular behavior. Soak time for each deployment was approximately $90 \mathrm{~min}$. BRUVS were equipped with high-definition (HD) GoPro Hero 3+ action cameras and baited with $1,000 \mathrm{~g}$ of a mix of crushed sardine and fish offal. Only the windward (northwestern) side of FN was sampled due to its calmer sea conditions at this time of the year.

\section{Video and data analysis}

All video samples were analyzed using the free VLC media player software (www.videolan.org). All fish were identified to species level, with few exceptions identified to genus level (e.g. Halichoeres spp.). 
The maximum number of individuals within a paused still frame at any time of the video (MaxN) was adopted as relative abundance measure, in order to avoid recounting individual entering several times in the camera's field of view (Cappo et al., 2004). Total relative abundances (TMaxN), summed from all BRUVS samples, per species are presented in a matrix (Table 1) with information on the international and Brazilian conservation status (IUCN, 2019; ICMBio, 2018.
Mean relative abundance and species richness per BRUVS deployment was also calculated. Four habitat types were visually identified: rocky reefs (RO), rocky bottom with macroalgae (MA) and rhodolith beds (RH), as well as the unconsolidated sandy bottom habitats (SA) (Figure 2). At last, species images were extracted from the video samples, in order to produce a catalog of underwater images of the species (Figures 3-28).

Table 1. Matrix of the fish inventory of Fernando de Noronha Archipelago obtained with Baited Remote Underwater Video Systems $(B R U V S)$. TMaxN = Total (summed) relative abundance; Conservation status according to IUCN $=$ The International Union for Conservation of Nature and ICMBio = Instituto Chico Mendes de Conservação da Biodiversidade; $\mathrm{CR}=$ Critically Endangered; VU = Vulnerable; NT = Near Threatened; LC = Least concern; DD = Data Deficient; $\mathrm{NE}=$ Not Evaluated. Benthic habitats: $\mathrm{SA}=$ Sandy bottom; RO = Rocky bottom / rocky reefs; MA = Rocky bottom with macroalgae and RH = Rhodolith

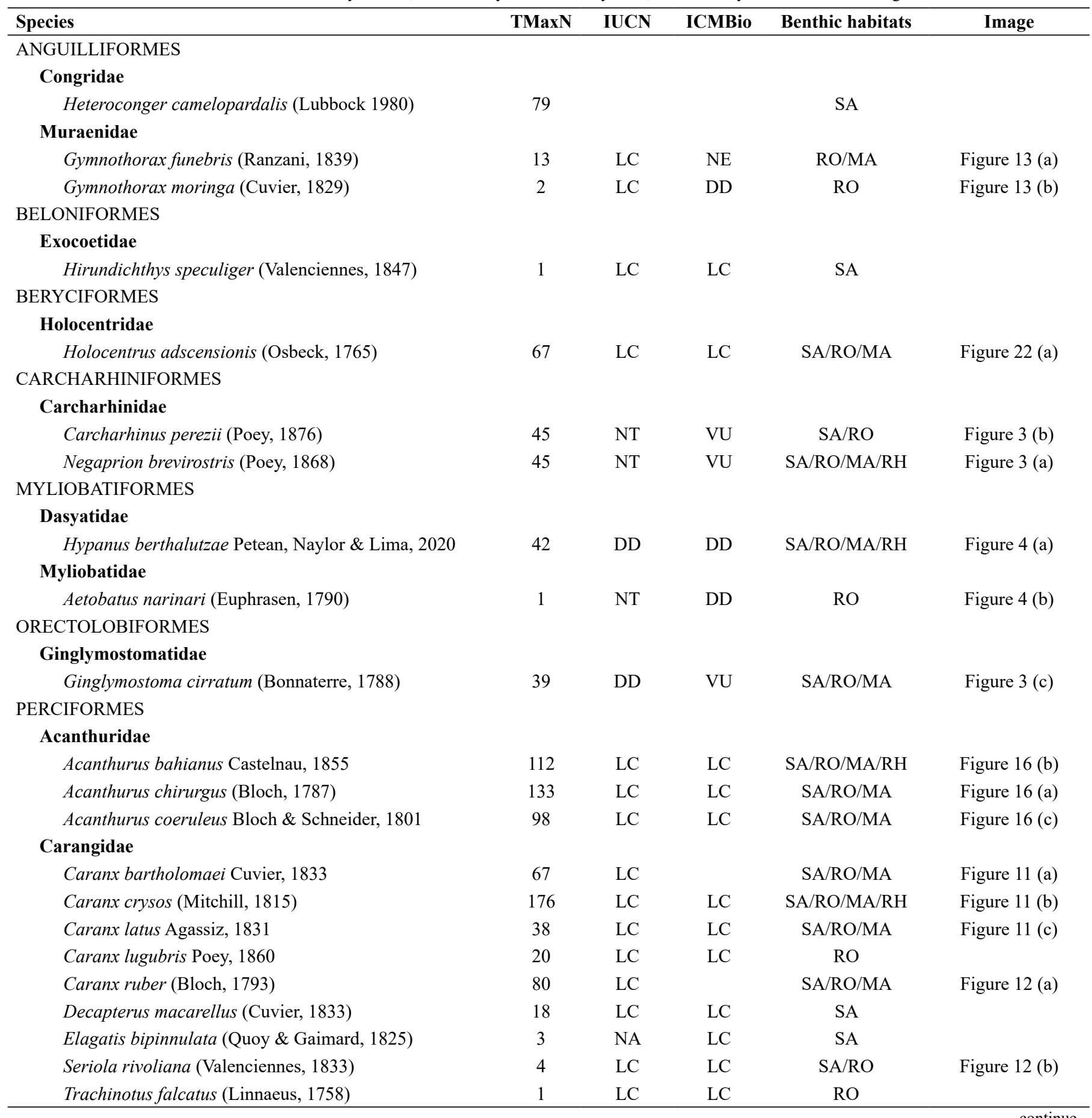




\begin{tabular}{|c|c|c|c|c|c|}
\hline Chaetodon ocellatus (Bloch, 1787) & 50 & $\mathrm{LC}$ & DD & $\mathrm{SA} / \mathrm{RO} / \mathrm{MA}$ & Figure 24 (b) \\
\hline Echeneis naucrates Linnaeus, 1758 & 44 & & $\mathrm{LC}$ & $\mathrm{SA} / \mathrm{RO} / \mathrm{MA}$ & Figure 27 (a) \\
\hline Echeneis neucratoides Zuiew, 1789 & 1 & DD & & SA & \\
\hline Anisotremus surinamensis (Bloch, 1791) & 107 & DD & DD & $\mathrm{SA} / \mathrm{RO} / \mathrm{MA} / \mathrm{RH}$ & Figure $14(a)$ \\
\hline Haemulon chrysargyreum (Günther, 1859) & 117 & $\mathrm{LC}$ & $\mathrm{LC}$ & $\mathrm{RO} / \mathrm{MA}$ & Figure $14(b)$ \\
\hline Haemulon parra (Desmarest, 1823) & 98 & $\mathrm{LC}$ & $\mathrm{LC}$ & $\mathrm{SA} / \mathrm{RO} / \mathrm{MA} / \mathrm{RH}$ & Figure $14(\mathrm{c})$ \\
\hline \multicolumn{6}{|l|}{ Kyphosidae } \\
\hline Kyphosus bigibbus Lacepède, 1801 & 104 & $\mathrm{LC}$ & & $\mathrm{SA} / \mathrm{RO} / \mathrm{MA} / \mathrm{RH}$ & Figure 15 (a) \\
\hline Bodianus rufus (Linnaeus, 1758) & 5 & $\mathrm{LC}$ & $\mathrm{LC}$ & RO & \\
\hline Clepticus brasiliensis Heiser, Moura \& Robertson, 2000 & 1 & $\mathrm{LC}$ & $\mathrm{LC}$ & RO & \\
\hline Halichoeres dimidiatus (Agassiz, 1831) & 30 & $\mathrm{LC}$ & & $\mathrm{SA} / \mathrm{RO}$ & \\
\hline Halichoeres radiatus (Linnaeus, 1758) & 83 & $\mathrm{LC}$ & $\mathrm{LC}$ & $\mathrm{SA} / \mathrm{RO} / \mathrm{MA}$ & Figure $18(b)$ \\
\hline Halichoeres spp. & 138 & & & $\mathrm{SA} / \mathrm{RO} / \mathrm{MA} / \mathrm{RH}$ & \\
\hline Xyrichtys incandescens (Linnaeus, 1758) & 1 & $\mathrm{LC}$ & $\mathrm{LC}$ & $\mathrm{SA}$ & \\
\hline \multicolumn{6}{|l|}{ Lutjanidae } \\
\hline Lutjanus jocu (Bloch \& Schneider, 1801) & 74 & DD & NT & $\mathrm{SA} / \mathrm{RO} / \mathrm{MA} / \mathrm{RH}$ & Figure 7 (a) \\
\hline \multicolumn{6}{|l|}{ Malacanthidae } \\
\hline \multicolumn{6}{|l|}{ Pomacentridae } \\
\hline Abudefduf saxatilis (Linnaeus, 1758) & 613 & hLC & $\mathrm{LC}$ & $\mathrm{SA} / \mathrm{RO} / \mathrm{MA} / \mathrm{RH}$ & Figure 17 (a) \\
\hline Chromis multilineata (Guichenot, 1853) & 263 & LC & LC & $\mathrm{SA} / \mathrm{RO} / \mathrm{MA}$ & Figure 17 (b) \\
\hline Stegastes pictus (Castelnau, 1855) & 34 & & $\mathrm{LC}$ & $\mathrm{SA} / \mathrm{RO} / \mathrm{MA}$ & \\
\hline Stegastes rocasensis ${ }^{\mathrm{E}}$ (Emery, 1972) & 15 & & VU & $\mathrm{RO} / \mathrm{MA}$ & Figure 17 (c) \\
\hline \multicolumn{6}{|l|}{ Scaridae } \\
\hline Sparisoma amplum (Ranzani, 1841) & 43 & $\mathrm{LC}$ & NT & $\mathrm{SA} / \mathrm{RO}$ & Figure $9(a, b)$ \\
\hline Sparisoma axillare (Steindachner, 1878) & 45 & DD & VU & $\mathrm{SA} / \mathrm{RO} / \mathrm{MA} / \mathrm{RH}$ & Figure 7 (b) \\
\hline Sparisoma frondosum (Agassiz, 1831) & 26 & DD & VU & $\mathrm{SA} / \mathrm{RO} / \mathrm{MA} / \mathrm{RH}$ & Figure 8 (a) \\
\hline Sparisoma sp. & 6 & & & $\mathrm{SA} / \mathrm{RO}$ & \\
\hline \multicolumn{6}{|l|}{ Serranidae } \\
\hline Cephalopholis fulva (Linnaeus, 1758) & 254 & $\mathrm{LC}$ & $\mathrm{LC}$ & $\mathrm{SA} / \mathrm{RO} / \mathrm{MA} / \mathrm{RH}$ & Figure 5 (a) \\
\hline Dermatolepis inermis (Valenciennes, 1833) & 27 & DD & $\mathrm{DD}$ & $\mathrm{SA} / \mathrm{RO}$ & Figure $6(b)$ \\
\hline Epinephelus itajara (Lichtenstein, 1822) & 1 & VU & $\mathrm{CR}$ & $\mathrm{RO}$ & Figure 5 (b) \\
\hline Paranthias furcifer (Valenciennes, 1828) & 108 & $\mathrm{LC}$ & & $\mathrm{SA} / \mathrm{RO} / \mathrm{MA}$ & Figure 6 (a) \\
\hline Rypticus saponaceus (Bloch \& Schneider, 1801) & 1 & $\mathrm{LC}$ & $\mathrm{LC}$ & RO & \\
\hline
\end{tabular}




\begin{tabular}{|c|c|c|c|c|c|}
\hline Species & TMaxN & IUCN & ICMBio & Benthic habitats & Image \\
\hline \multicolumn{6}{|l|}{ Sphyraenidae } \\
\hline Sphyraena barracuda (Edwards, 1771) & 68 & LC & LC & $\mathrm{SA} / \mathrm{RO} / \mathrm{MA} / \mathrm{RH}$ & Figure 10 (a) \\
\hline \multicolumn{6}{|l|}{ PLEURONECTIFORMES } \\
\hline \multicolumn{6}{|l|}{ Bothidae } \\
\hline Bothus lunatus (Linnaeus, 1758) & 1 & LC & LC & MA & Figure 26 (a) \\
\hline Bothus maculiferus (Poey, 1860) & 2 & $\mathrm{LC}$ & $\mathrm{LC}$ & $\mathrm{SA} / \mathrm{RO}$ & \\
\hline \multicolumn{6}{|l|}{ SCORPAENIFORMES } \\
\hline \multicolumn{6}{|l|}{ Dactylopteridae } \\
\hline Dactylopterus volitans (Linnaeus, 1758) & 16 & LC & $\mathrm{LC}$ & SA/MA & Figure 28 (a) \\
\hline \multicolumn{6}{|l|}{ TETRAODONTIFORMES } \\
\hline \multicolumn{6}{|l|}{ Balistidae } \\
\hline Canthidermis sufflamen (Mitchill, 1815) & 15 & LC & LC & $\mathrm{SA} / \mathrm{RO} / \mathrm{MA}$ & \\
\hline Melichthys niger (Bloch, 1786) & 628 & LC & LC & $\mathrm{SA} / \mathrm{RO} / \mathrm{MA}$ & Figure 19 (a) \\
\hline \multicolumn{6}{|l|}{ Monacanthidae } \\
\hline Aluterus scriptus (Osbeck, 1765) & 5 & LC & LC & $\mathrm{RO} / \mathrm{SA} / \mathrm{MA}$ & Figure 20 (a) \\
\hline Cantherhines macrocerus (Hollard, 1853) & 9 & LC & LC & $\mathrm{SA} / \mathrm{RO} / \mathrm{MA}$ & Figure $20(b)$ \\
\hline \multicolumn{6}{|l|}{ Ostraciidae } \\
\hline Lactophrys trigonus (Linnaeus, 1758) & 61 & $\mathrm{LC}$ & $\mathrm{LC}$ & $\mathrm{SA} / \mathrm{RO} / \mathrm{MA}$ & Figure 24 (a) \\
\hline
\end{tabular}

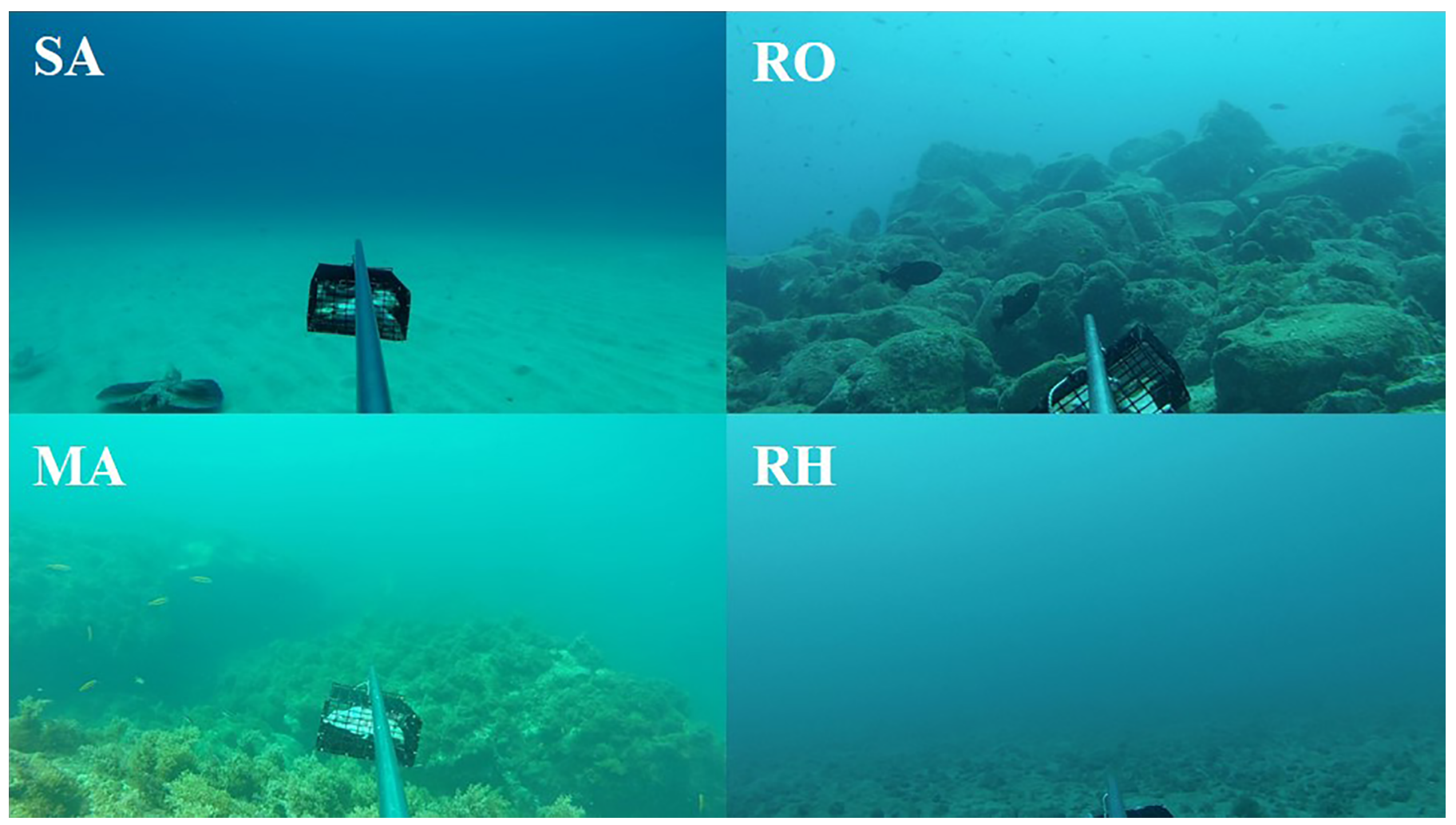

Figure 2. Benthic habitat types visually identified during video analysis from BRUVS (Baited Remote Underwater Video System) samples at the Fernando de Noronha Archipelago, Brazil. SA = Sandy bottom; RO = Rocky bottom $/$ rocky reefs; MA = Rocky bottom with macroalgae and $\mathrm{RH}=\mathrm{Rhodolith}$ 
Schmid, K. et al.

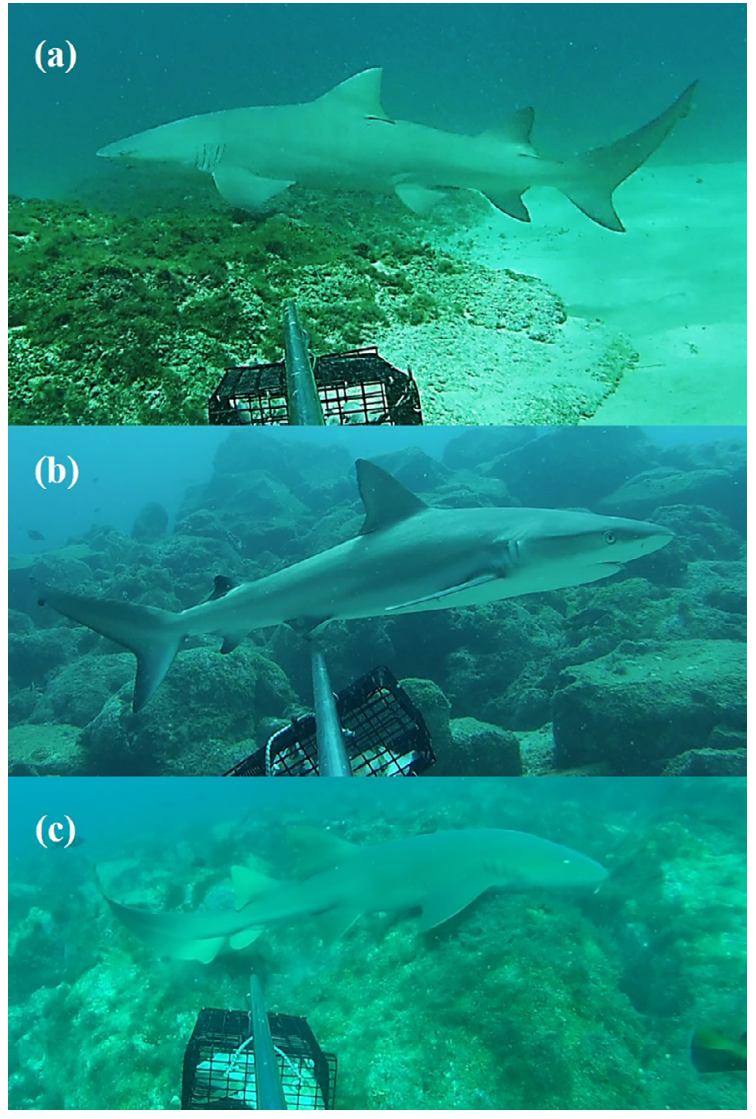

Figure 3. (a) Negaprion brevirostris; (b) Carcharhinus perezii; (c) Ginglymostoma cirratum

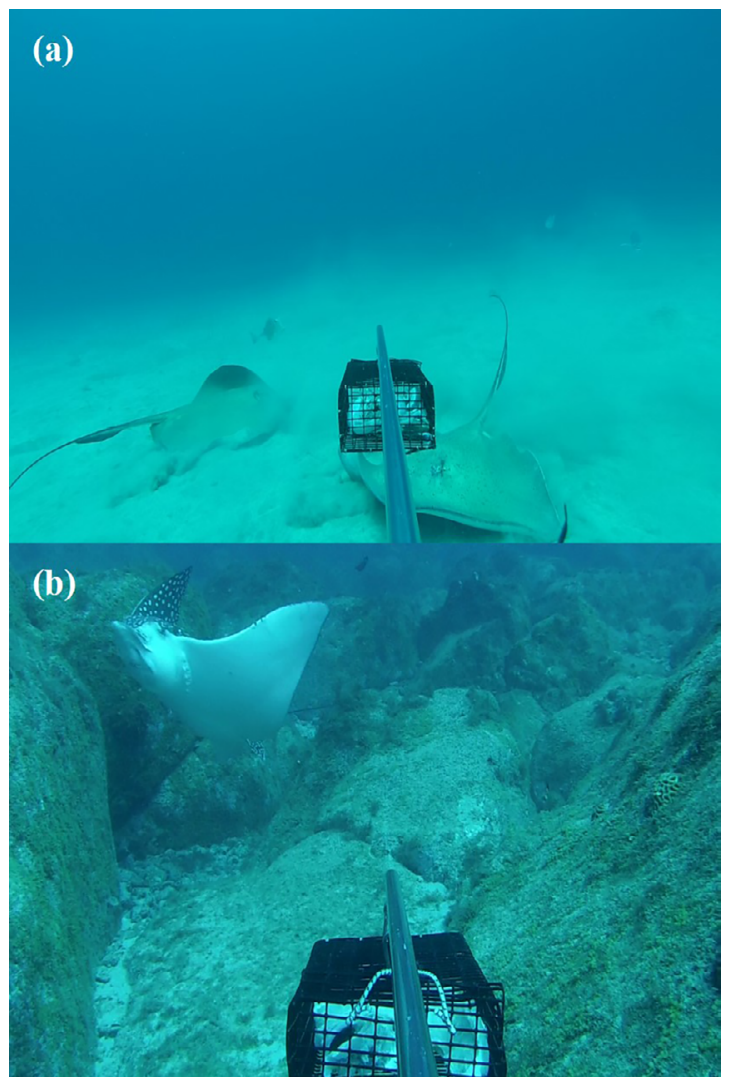

Figure 4. (a) Hypanus americanus; (b) Aetobatus narinari

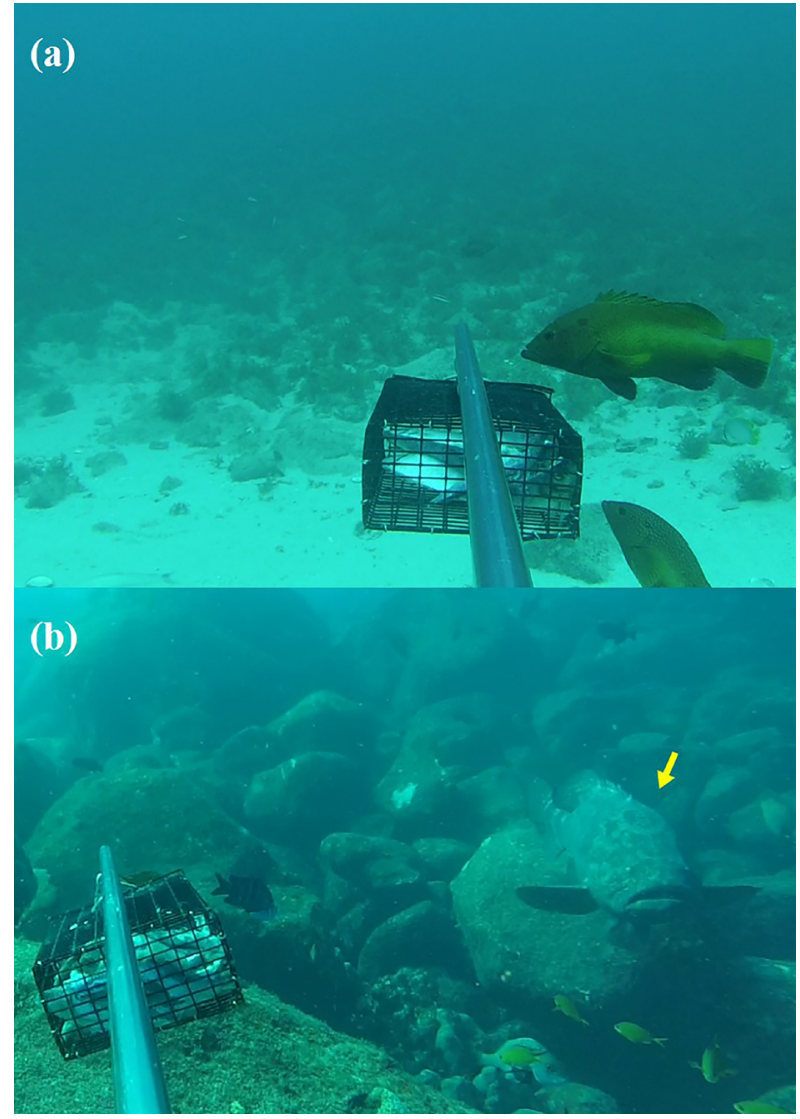

Figure 5. (a) Cephalopholis fulva; (b) Epinephelus itajara

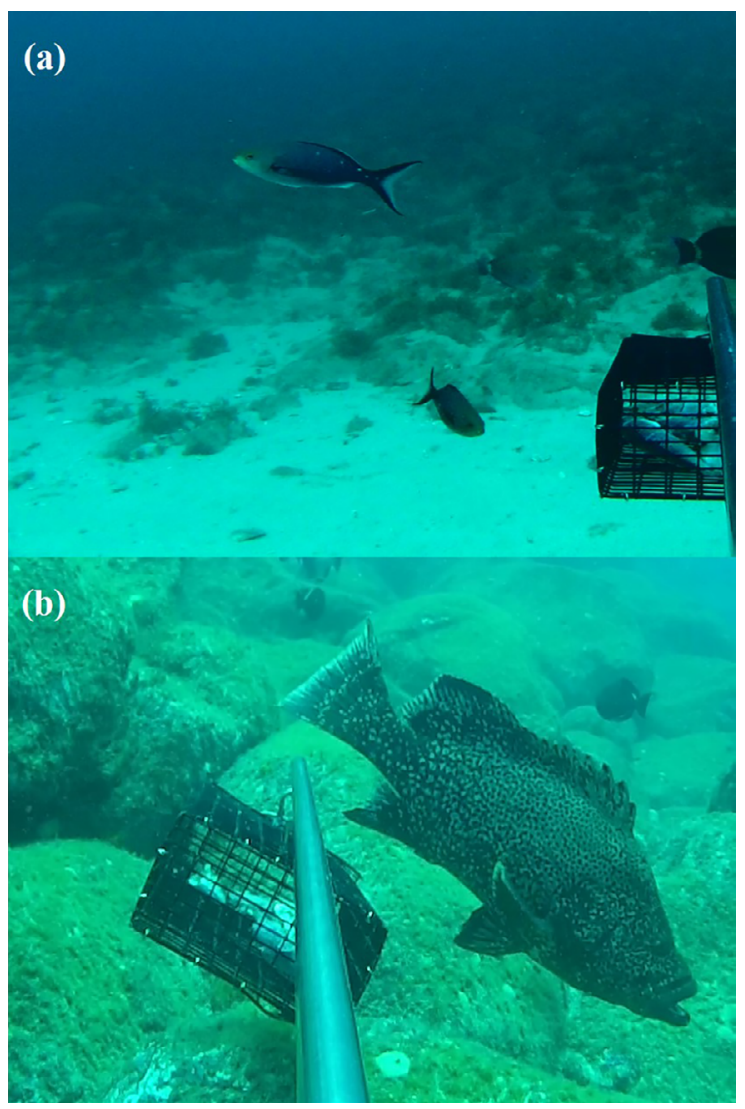

Figure 6. (a) Paranthias furcifer; (b) Dermatolepis inermis

https://doi.org/10.1590/1676-0611-BN-2020-1014 


\section{(a)}

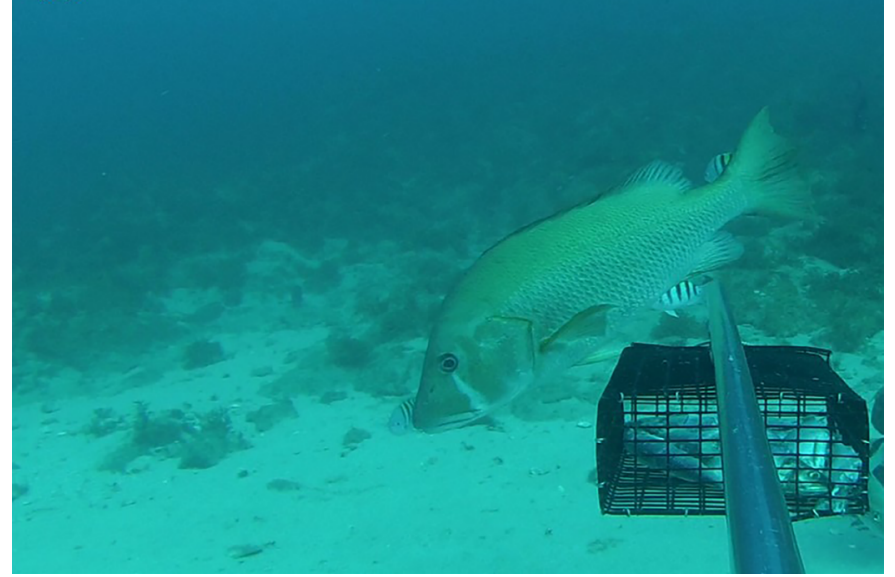

Figure 7. (a) Lutjanus jocu

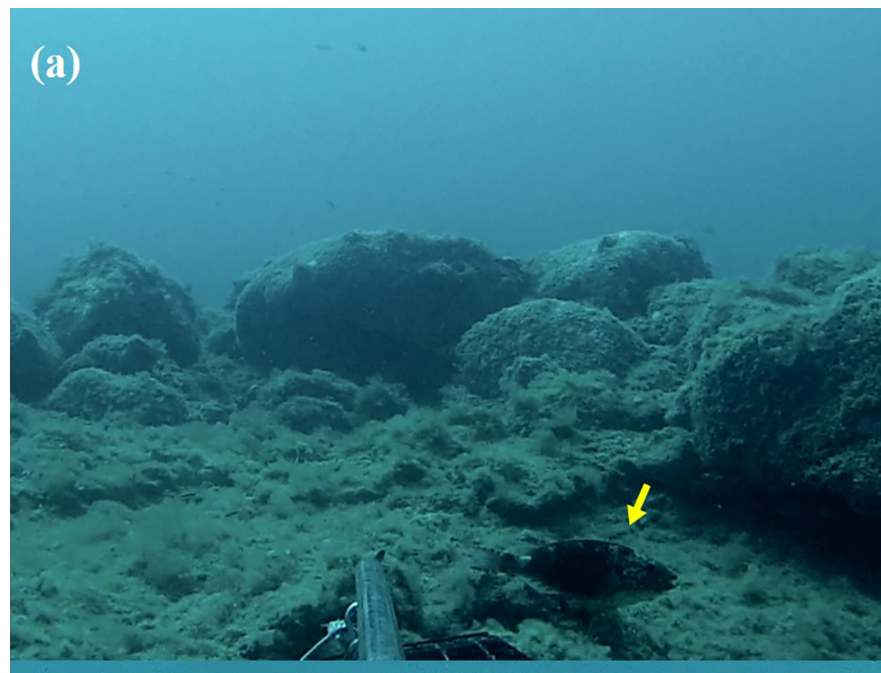

(b)

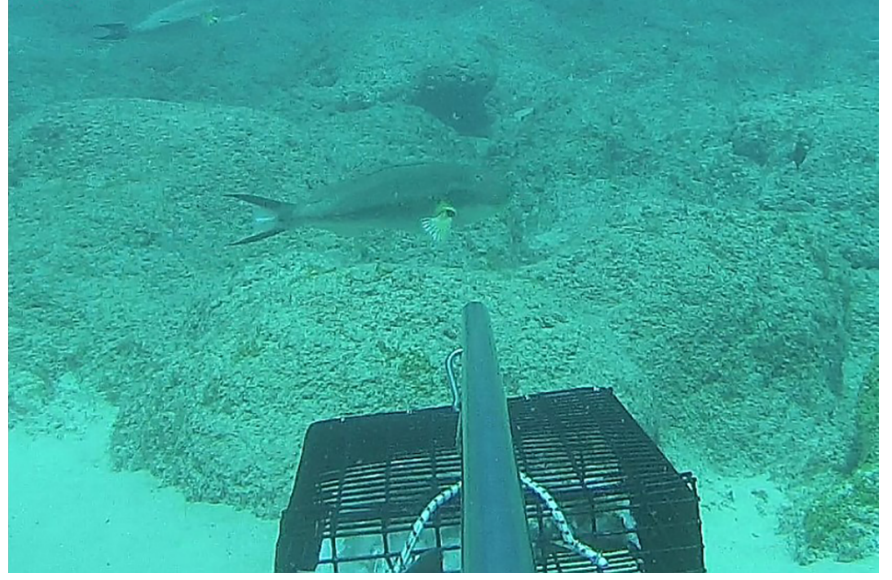

Figure 8. (a) Sparisoma frondosum (intermediate phase); (b) Sparisoma axillare (terminal phase)
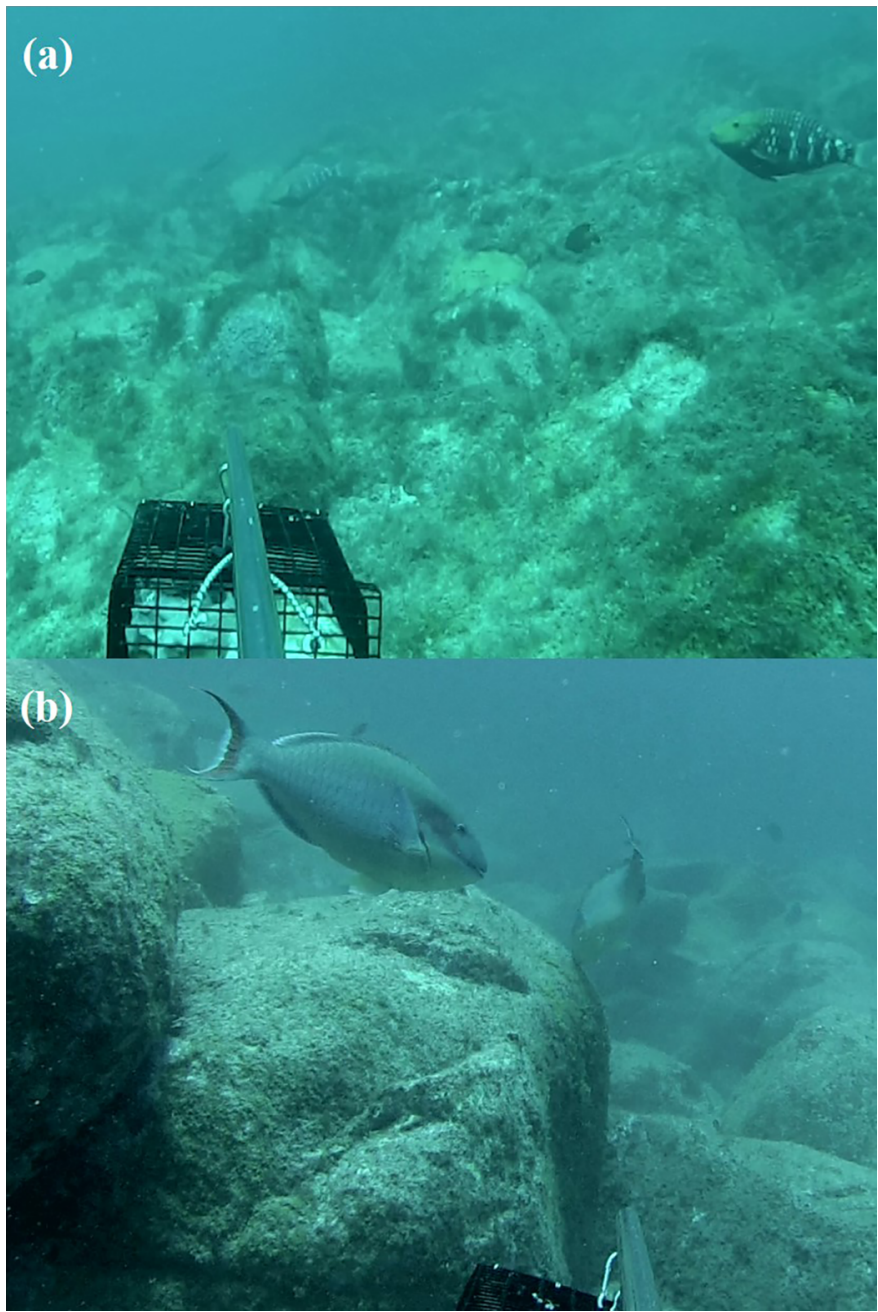

Figure 9. (a) Sparisoma amplum (intermediate phase); (b) Sparisoma amplum (terminal phase)

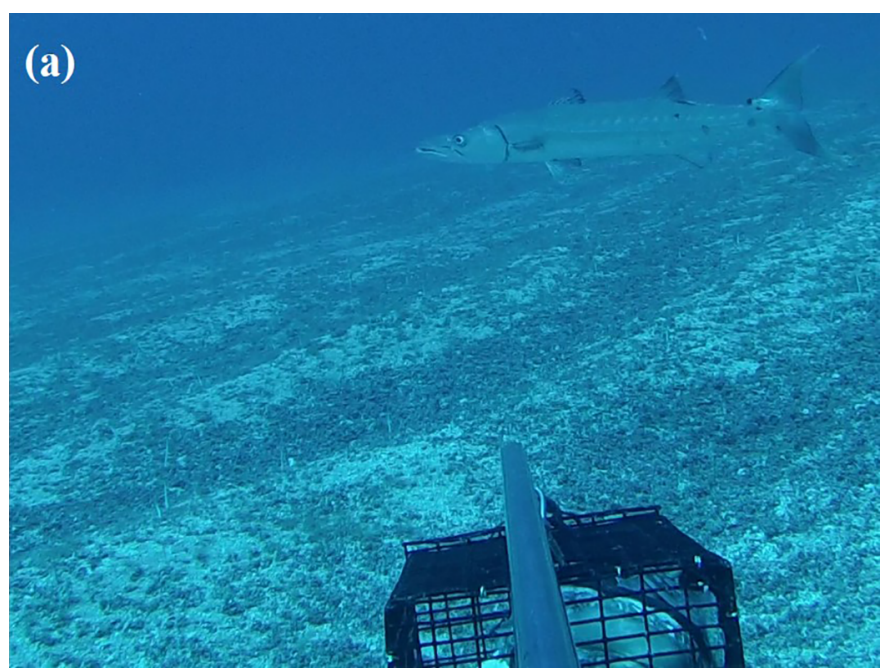

Figure 10. (a) Sphyraena barracuda 
Schmid, K. et al.

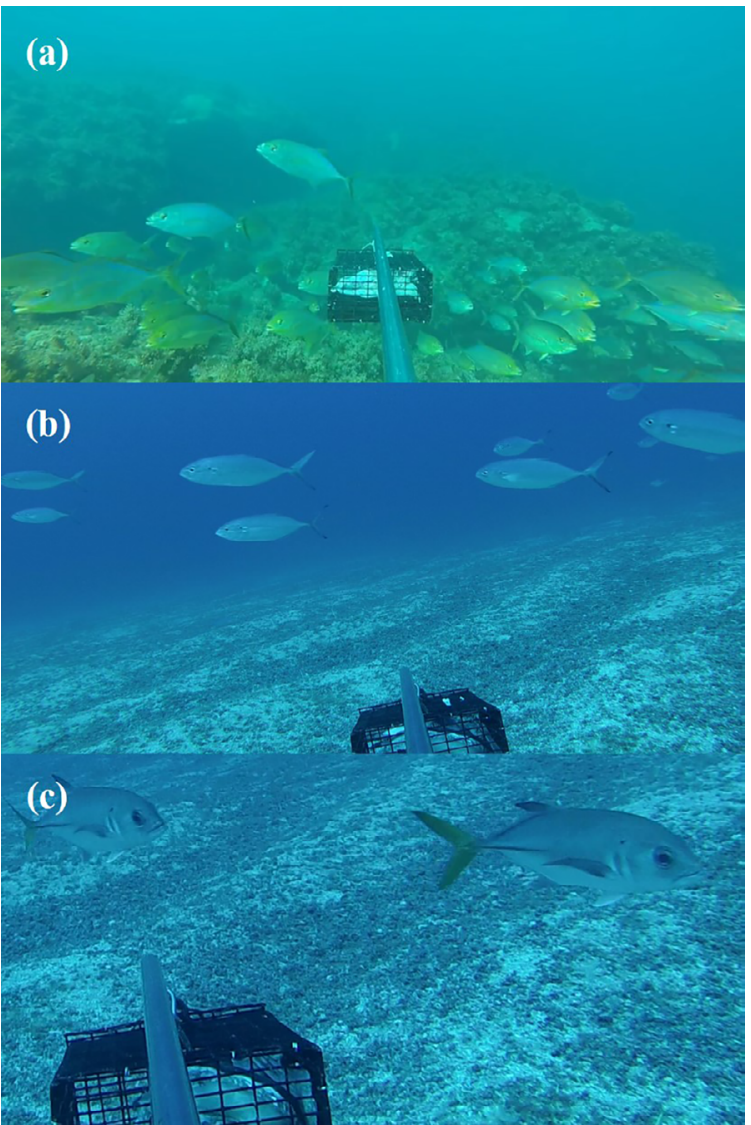

Figure 11. (a) Caranx bartholomaei; (b) Caranx crysos; (c) Caranx latus

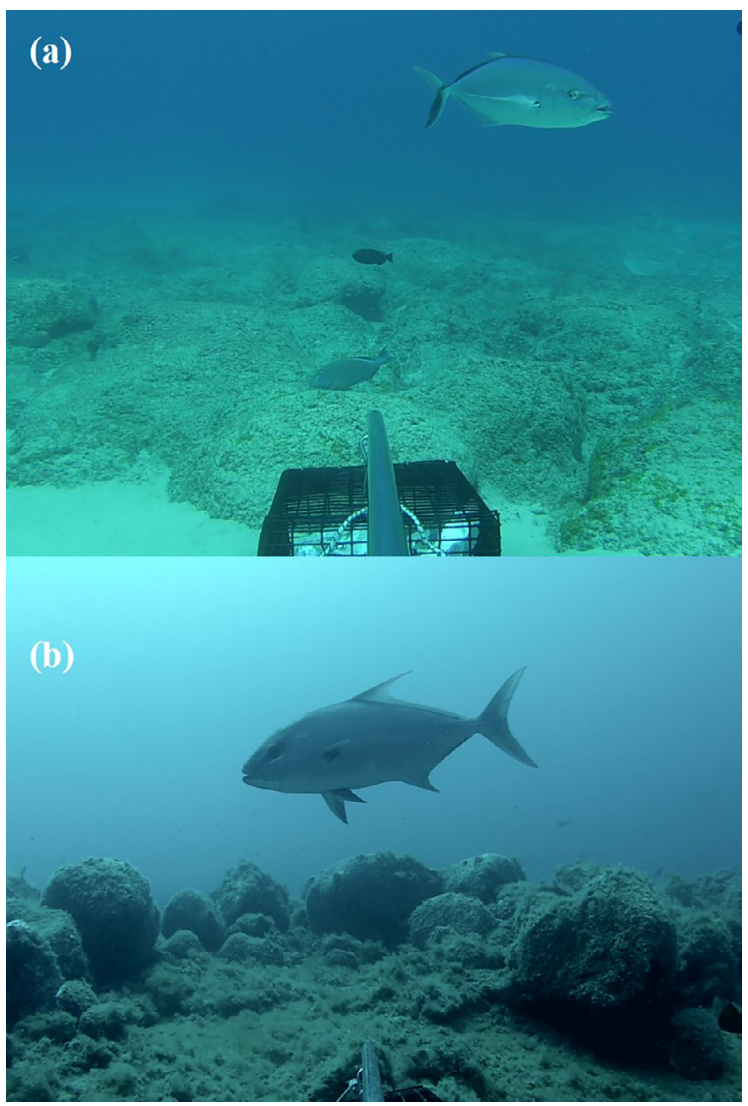

Figure 12. (a) Caranx ruber; (b) Seriola rivoliana

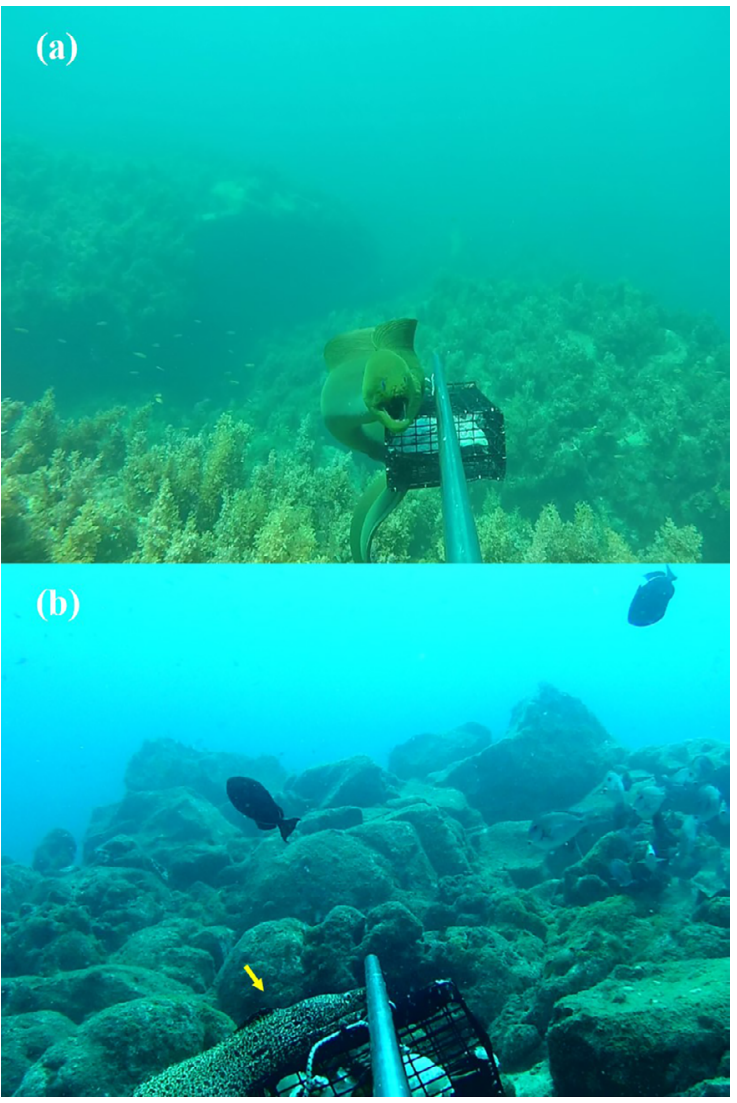

Figure 13. (a) Gymnothorax funebris; (b) Gymnothorax moringa

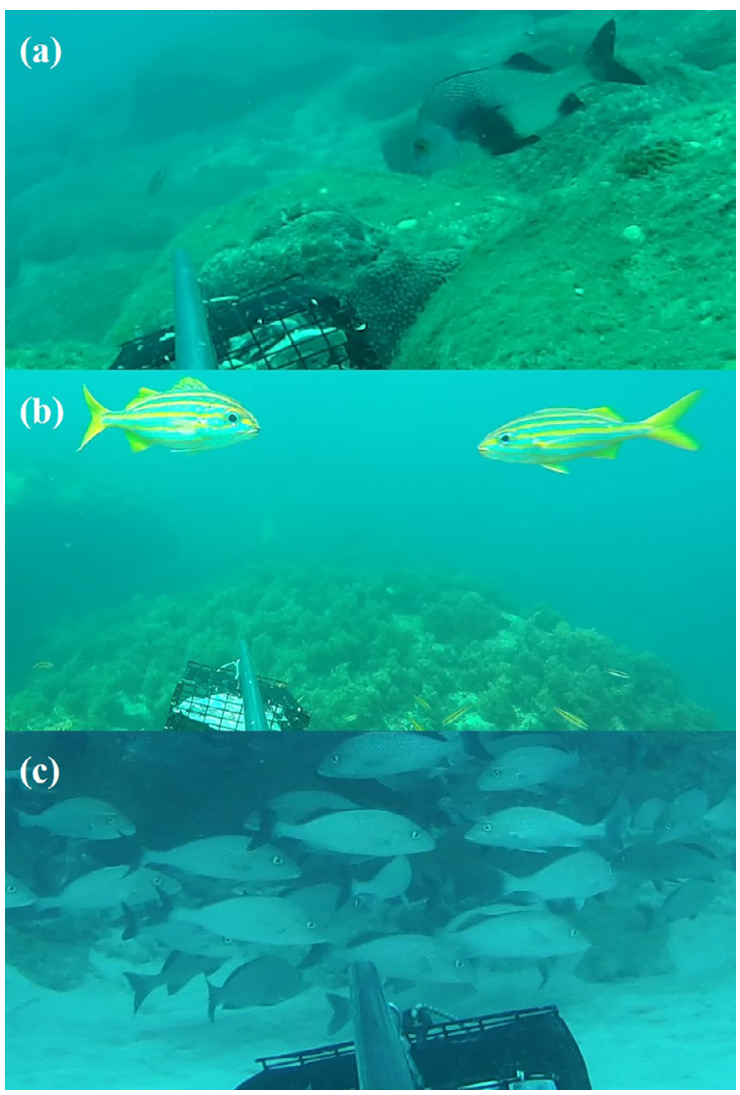

Figure 14. (a) Anisotremus surinamensis; (b) Haemulon chrysargyreum ; (c) Haemulon parra 


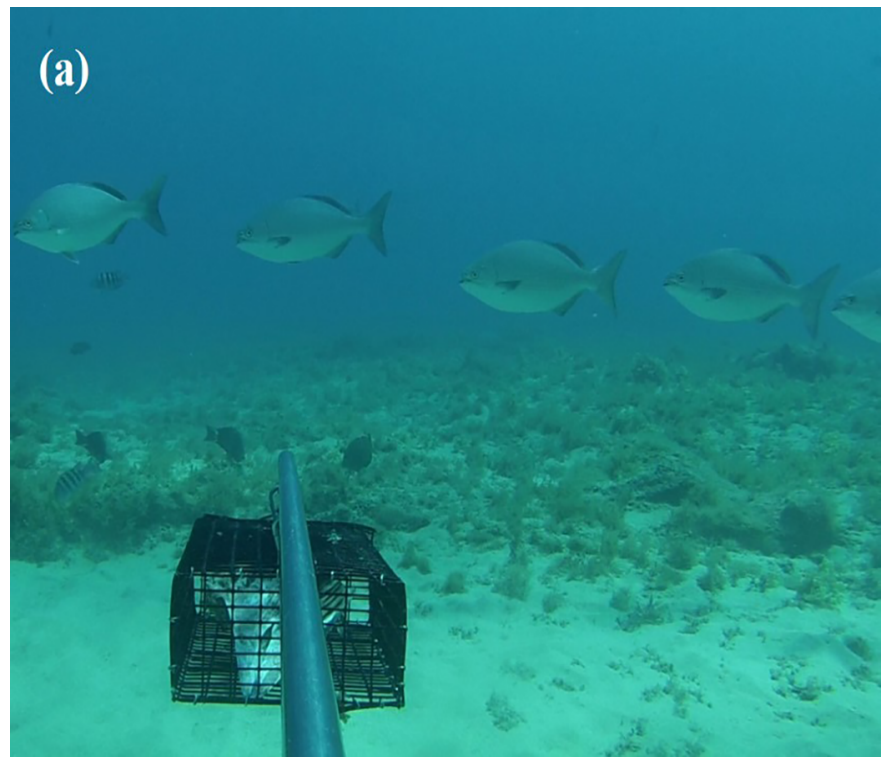

Figure 15. (a) Kyphosus bigibbus

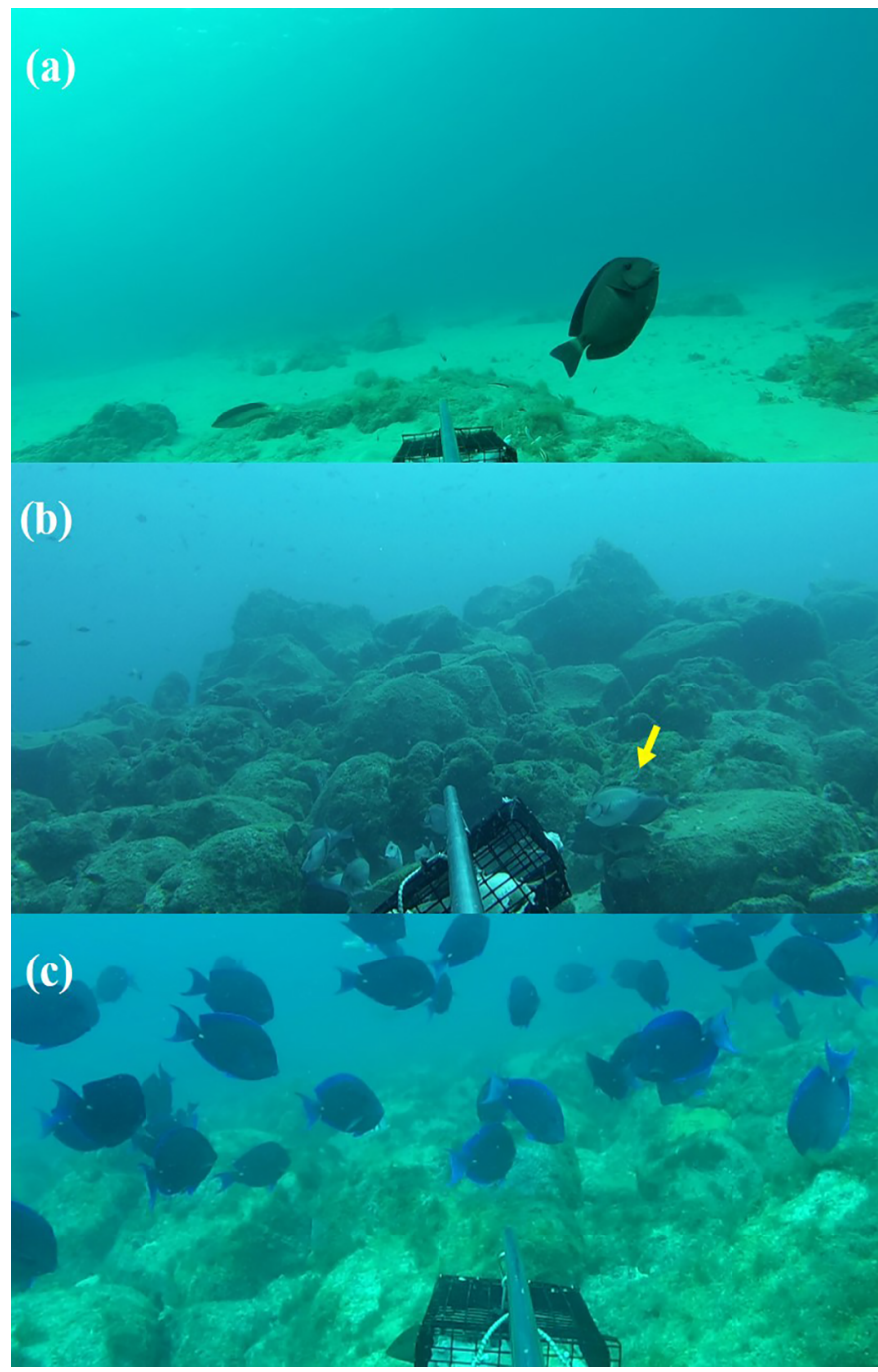

Figure 16. (a) Acanthurus bahianus; (b) Acanthurus chirurgus; (c) Acanthurus coeruleus

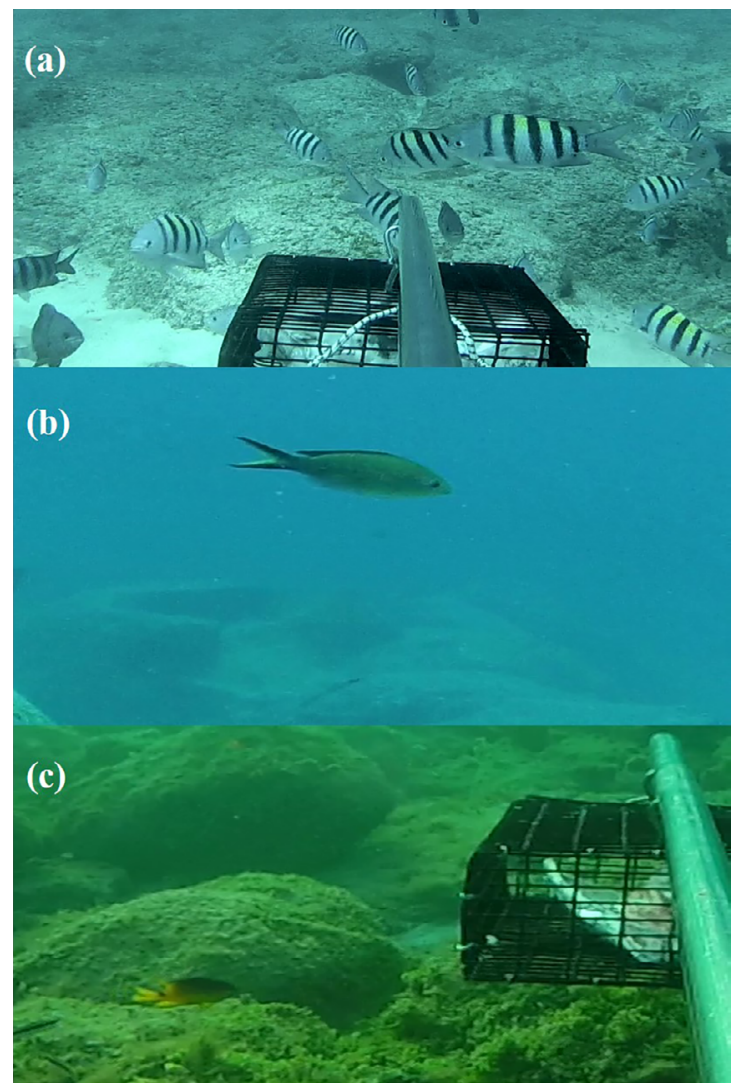

Figure 17. (a) Abudefduf saxatilis; (b) Chromis multilineata; (c) Stegastes rocasensis

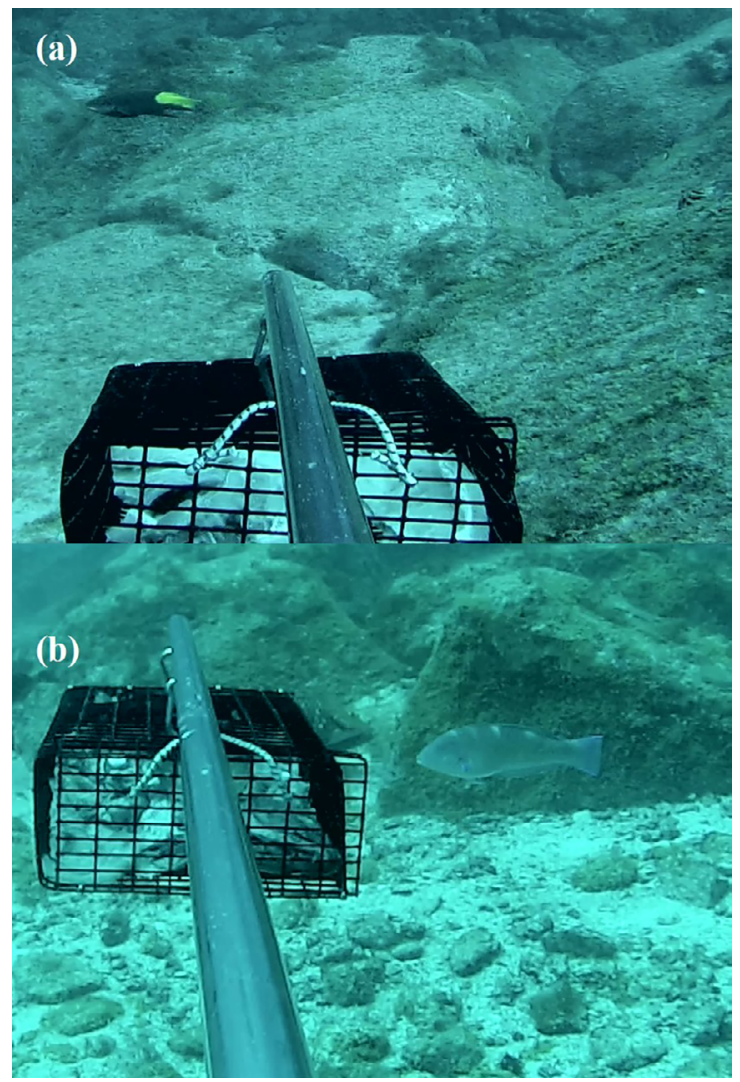

Figure 18. (a) Bodianus pulchellus; (b) Halichoeres radiatus (terminal phase) 
Schmid, K. et al.

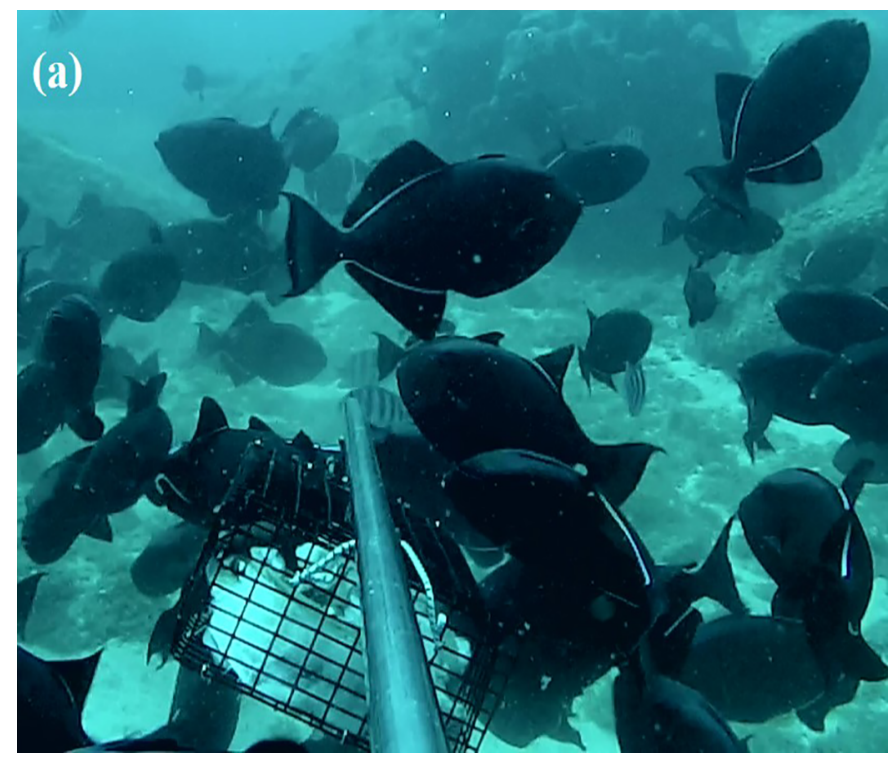

Figure 19. (a) Melichthys niger

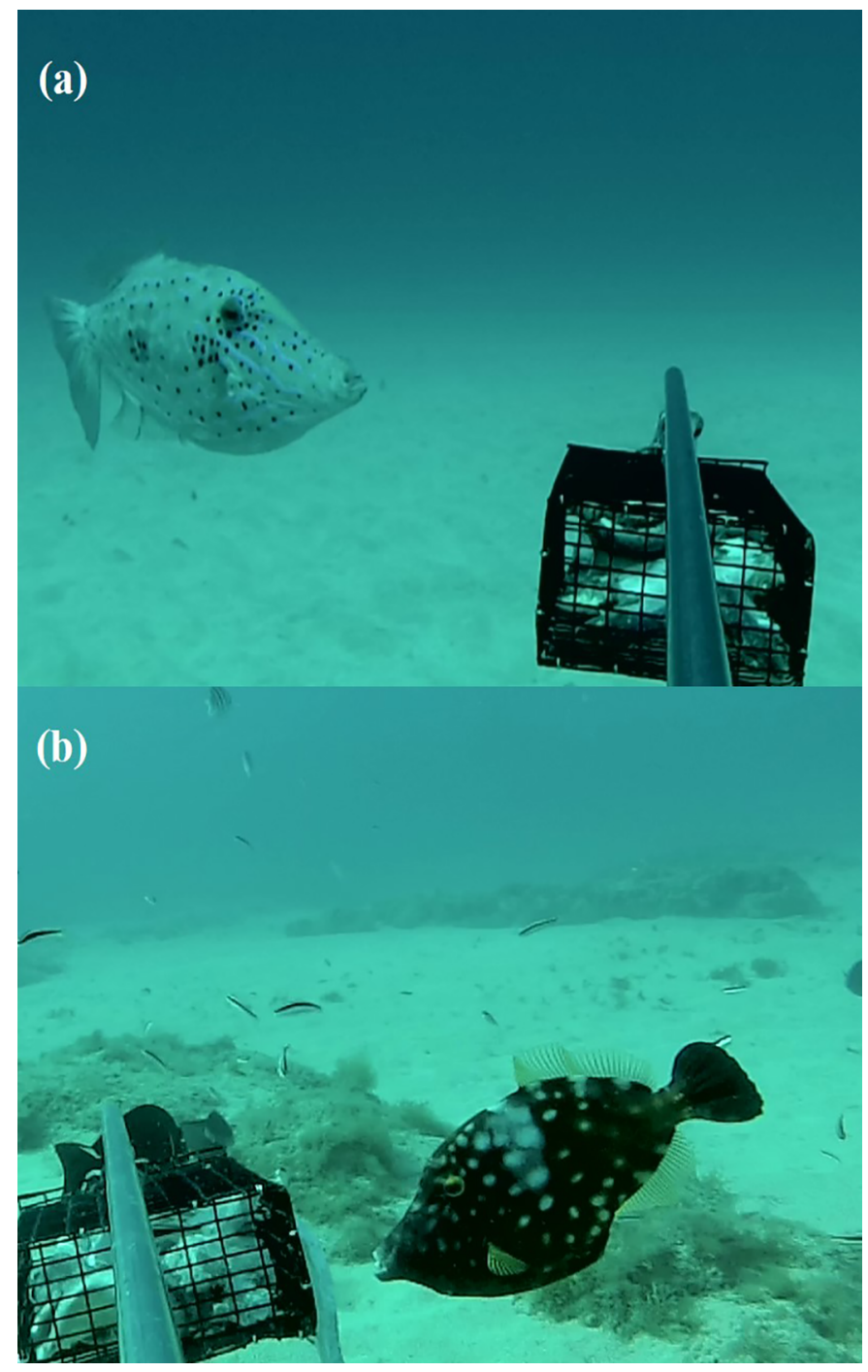

Figure 20. (a) Aluterus scriptus; (b) Cantherhines macrocerus

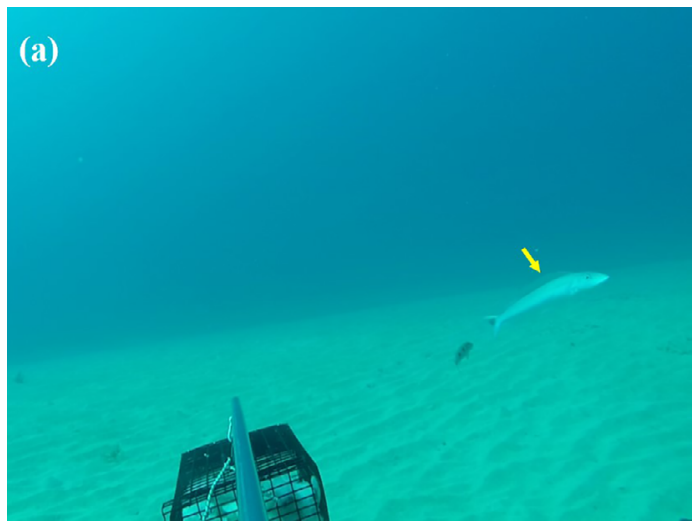

Figure 21. (a) Malacanthus plumieri

(a)

\section{Figure 22. (a) Holocentrus adscensionis}

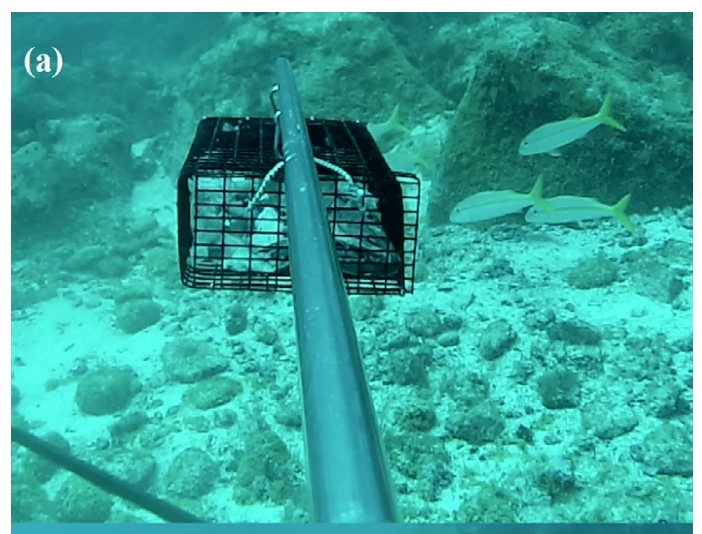

(b)

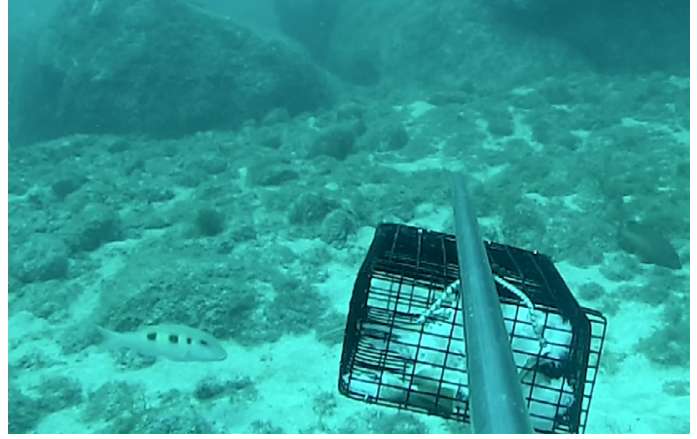

Figure 23. (a) Mulloidichthys martinicus; (b) Pseudupeneus maculatus 
Fernando de Noronha fish inventory

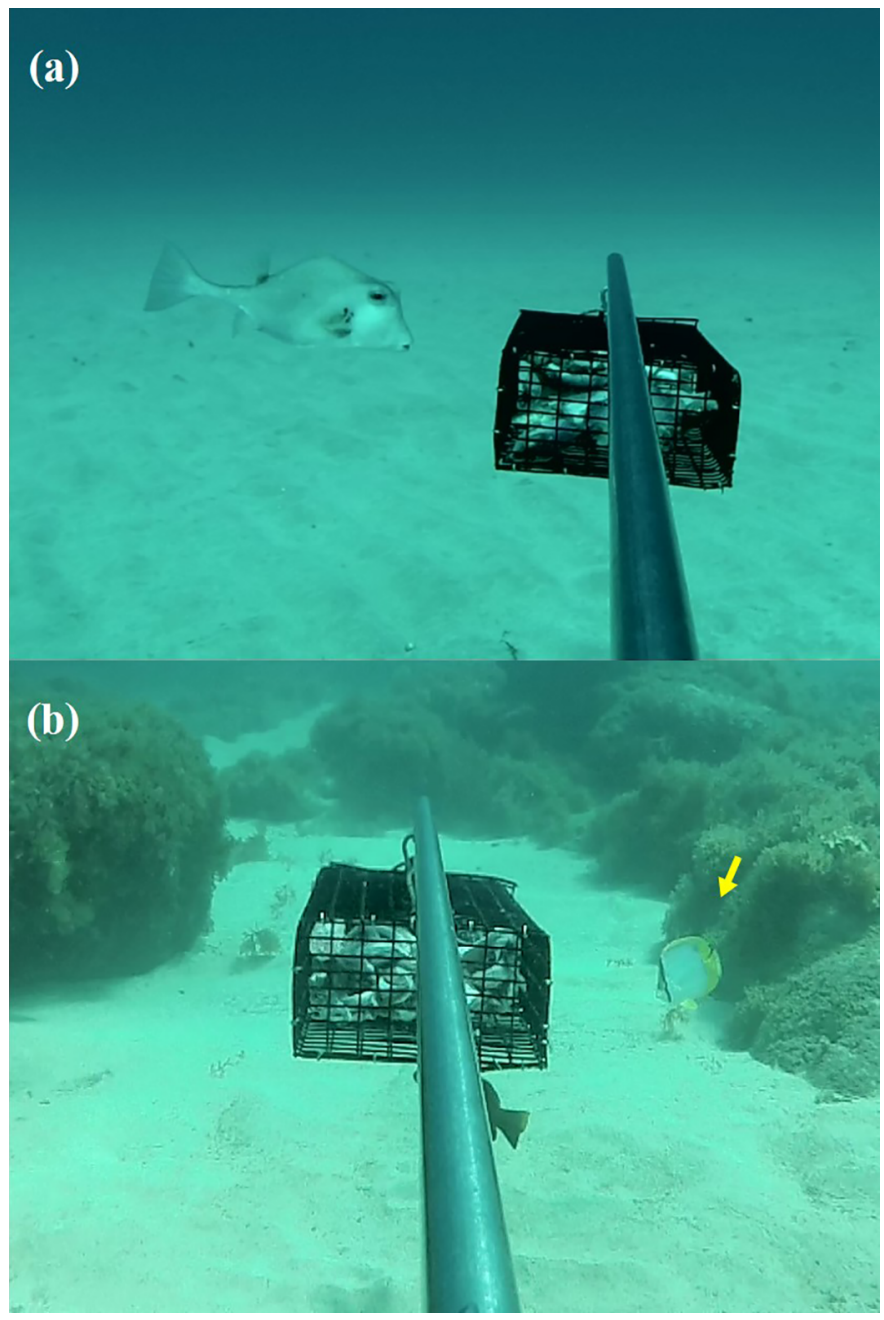

Figure 24. (a) Lactophrys trigonus; (b) Chaetodon ocellatus

\section{(a)}

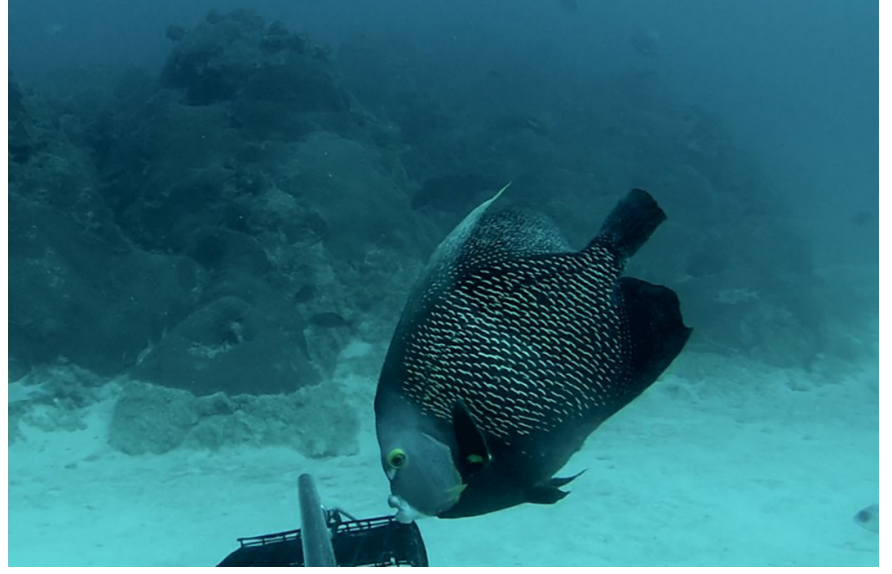

Figure 25. (a) Pomacanthus paru

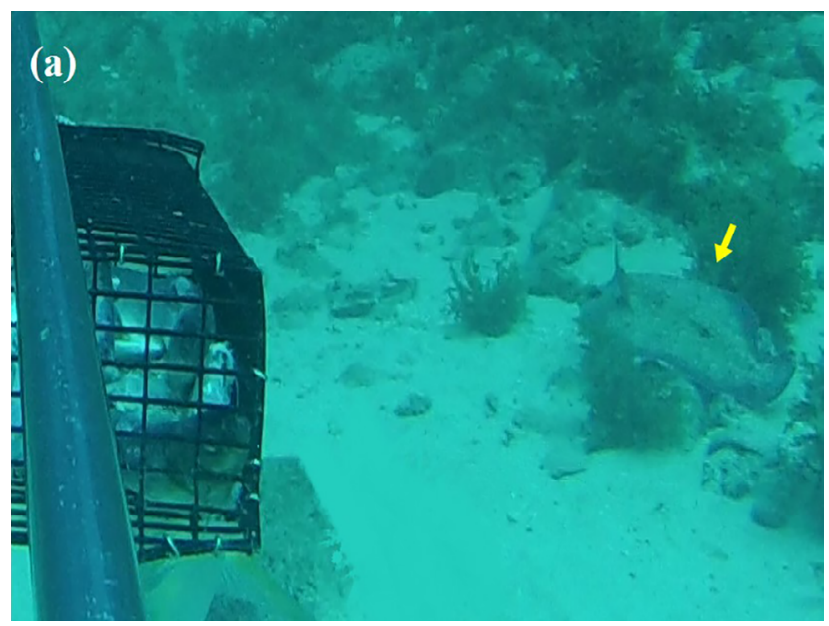

Figure 26. (a) Bothus lunatus

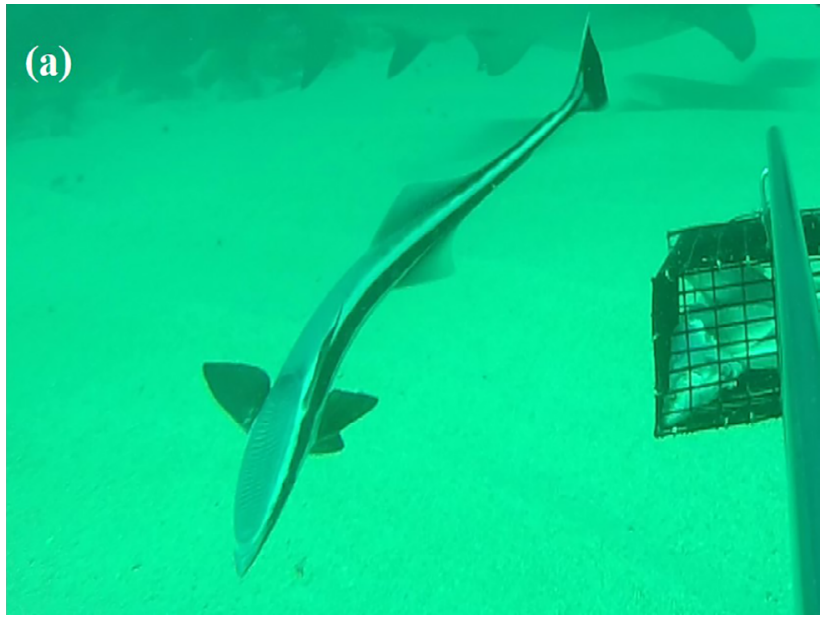

Figure 27. (a) Echeneis naucrates

(a)

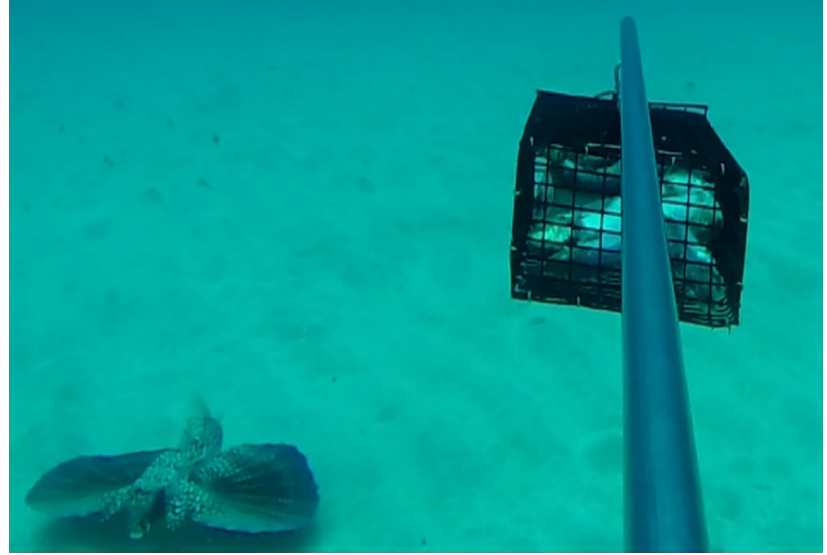

Figure 28. (a) Dactylopterus volitans 


\section{Results and Discussion}

From the 81 BRUVS deployments, 4,398 individuals of 66 fish species, belonging to 28 families and 10 orders were identified. The overall mean relative abundance (mean MaxN $\pm \mathrm{SD}$ ) and species richness (mean $\mathrm{S} \pm \mathrm{SD}$ ) sampled per BRUVS deployment were $54.3 \pm 46.2$ and $13.9 \pm 8.8$, respectively. In the rocky reefs, the mean relative abundance and species richness were $96.4 \pm 38.2$ and $22.5 \pm 5.1$, in rocky bottom with macroalgae $65.8 \pm 39.8$ and $17.6 \pm 9.7$, in rhodolith beds $32.5 \pm 40.3$ and 7.5 \pm 6.4 and in sandy bottom habitats $25.3 \pm 25.9$ and $8.0 \pm 5.3$, respectively. BRUVS were deployed randomly, without prior identification of the habitat types at the sampling locations, which contributed to a highly unbalanced number of samples per habitat (SA: 44/ RO: 30 / MA: 5 / RH: 2), unsuitable for further statistical analysis and testing, yet still serving as an additional information, that may support future species-specific or habitatrelated studies in the Archipelago.

Compared to former fish fauna inventories in this area (Soto, 2011; Krajewski \& Floeter, 2011; Medeiros et al., 2011; Ilarri et al., 2017; Andrades et al., 2018), a considerably high number of species (66) was detected within the 81 BRUVS samples. Krajewski \& Floeter (2011) detected 60 species in 91 belt transects with scuba, sampling both sides of FN and the same depth range as this study. Ilari et al. (2017) recorded 50 species from 27 families in a study employing an effort of 140 transects in waters up to $6 \mathrm{~m}$ depth. Soto (2001) recorded 167 species, including pelagic species in an inventory using visual census and fisheries surveys supplemented with literature records. Furthermore, seven species of six different families have been recorded with BRUVS in this study, which were not registered in the former, above mentioned, studies. Being them Acanthurus bahianus (Acanthuridae), Bothus maculiferus (Bothidae), Caranx ruber and Trachinotus falcatus (both Carangidae), Hirundichthys speculiger (Exocoetidae), Clepticus brasiliensis and Halichoeres dimidiatus (Labridae).

Based on these results, BRUVS have shown to be a valuable alternative sampling method, that may eventually complement or even partially substitute traditionally used methods, in FN and other tropical marine protected areas, depending on which portion of the fish assemblage is studied. In this study, notably large predatory fish such as sharks, groupers and snappers were registered in high abundances, e.g. Carcharinus perezii and Negaprion brevirostris, each with 45 individuals, highlighting the efficiency of BRUVS to sample large-bodied, olfactory driven predatory fish. Studies developed with nonlethal methods are especially recommended for research on elasmobranchs, as this group includes some of the most vulnerable marine species (Musick et al., 2000).

The most representative families were Carangidade (407 individuals / 9 species), followed by Serranidae ( 385 individuals / 5 species), Labridae (272 individuals / 7 species) and Scaridae (120 individuals / 5 species).
Twelve families were represented by a single species (Table 1). The most abundant species were Melichthys niger (TMaxN: 628), Abudefduf saxatilis (TMaxN: 613), Chromis multilineata (TMaxN: 263) and Cephalopholis fulva (TMaxN: 254). For 10 species only a single individual was recorded in all video samples (Table 1). Furthermore, five different species of elasmobranchs of three orders and four families were also recorded, being them: Carcharinus perezii, Negaprion brevirostris, Ginglymostoma cirratum, Aetobatus narinari and Hypanus berthalutzae. All elasmobranchs showed high total relative abundances (TMaxN) ranging from 39 to 45 individuals (Table 1), except for one single individual of Aetobatus narinari (Myliobatiformes). From the 66 fish species recorded, a total of 49 species images were suitable to produce an underwater image species catalog (Figures 3-27). The specific image number for each species is indicated in the main matrix (Table 1).

Several threatened fish species were recorded, including the Goliath grouper Epinephelus itajara, classified as "Vulnerable" by the IUCN and already "Critically Endangered" by the Brazilian environmental agency (ICMBio). Three shark species classified as "Vulnerable" by the ICMBio, the Caribbean reef shark Carcharinus perezii, Lemon shark Negaprion brevirostris and the Nurse shark Ginglymostoma cirratum, were registered in large numbers, with 45, 45 and 39 individuals, respectively. Overall, more than $17 \%$ of all species registered in the present study are threatened (CR, VU) or near threatened (NT) according to IUCN and/or ICMBio (Table 1). The relatively high numbers of large predatory fish, threatened along most of the Brazilian coast, are certainly a positive response to three decades of protection from fishing by the PARNAMAR.

Finally, we recommend future research, ideally with stereoBRUVS that additionally enables fish length measurements, applied both in benthic and pelagic habitats of FN and surrounding areas. The information of species richness and relative abundance within FN provides a baseline for future monitoring efforts in order to evaluate the impact of long-term environmental changes. Yet, in order to achieve a much complete fish fauna inventory of FN, we still recommend employing a combination of BRUVS with other conventional sampling methods, to compensate for the BRUV's disadvantage in detecting, for instance, small cryptic living fish species.

\section{Acknowledgements}

Paul G. Allen Philanthropies/ Global FinPrint provided equipment and resources for the fieldwork. We are grateful to José Alexandre Carvalho and captain Roberto of the RV Capitania for logistical support, and Gina Clementi (Global FinPrint) for invaluable assistance in the field. This study was financed in part by the Coordenação de Aperfeiçoamento do Pessol de Nível Superior - Brasil (CAPES) - Finance Code 001 (K.S.). F.S. received a PIBIC scholarship from the Brazilian National Council for Scientific and Technological Development (CNPq). N.B. was funded by the Fundação de Amparo à Ciência e Tecnologia do Estado de Pernambuco (FACEPE). T.G. receives a productivity grant from CNPq 311078/2019-2. Our thanks to the editor and reviewers for the constructive comments and recommendations, which improved quality of the paper. 


\section{Author Contributions}

Kurt Schmid: Conceptualization, Formal analysis, Writing - original draft, Writing - review \& editing.

Fábio Renan Miranda da Silva: Formal analysis, Writing - review \& editing.

Bárbara Janaína Vieira dos Santos: Formal analysis, Writing review \& editing.

Natalia Priscila Alves Bezerra: Data collection, Formal analysis, Writing - review \& editing.

Ricardo Clapis Garla: Conceptualization, Data collection, Formal analysis, Writing - review \& editing, Funding acquisition.

Tommaso Giarrizzo: Conceptualization, Resources, Writing review \& editing, Funding acquisition, Academic supervision.

\section{Conflict of Interest}

The authors declare that they have no conflict of interest related to the publication of this manuscript.

\section{Ethics}

No approval by the ethics committee of the authors research institution was required, as the present study does not involve any manipulation of any living being whatsoever. All fish fauna data (video footage) were exclusively acquired by independent underwater video systems (BRUVS).

\section{Data availability}

The raw data of this study are very large data volumes of highresolution video footage and the summed data of the video analysis are already included in the submitted manuscript (Principal.doc)

\section{References}

ANDRADES, R., JOYEUX, J. C., ANDRADE, J. M., MACHADO, F. S., REISFILHO, J. A., MACIEIRA, R. M., \& GIARRIZZO, T. 2018. Filling the gap: Length-weight and length-length relationships of intertidal endemic fishes of the Brazilian Province Oceanic Islands. Journal of applied ichthyology, 34(3): 720-723.

CAPPO, M., HARVEY, E., MALCOLM, H., \& SPEARE, P. 2003. Potential of video techniques to monitor diversity, abundance and size of fish in studies of marine protected areas, in: Beumer, J.P., Grant, A., Smith, D.C. (Eds.), APAC Congress 2002: Aquatic protected areas - What works best and how do we know? World Congress on Aquatic Protected Areas proceedings. National Library of Australia, Cairns, Qld, Australia, pp. $455-464$.

DUDLEY, N. (Editor). 2008. Guidelines for Applying Protected Area Management Categories. Gland, Switzerland: IUCN. $x+86 p p$. WITH Stolton, S., P. Shadie and N. Dudley (2013). IUCN WCPA Best Practice Guidance on Recognising Protected Areas and Assigning Management Categories and Governance Types, Best Practice Protected Area Guidelines Series No. 21, Gland, Switzerland: IUCN

FLOETER, S. R., \& GASPARINI, J. L. 2000. The southwestern Atlantic reef fish fauna: composition and zoogeographic patterns. Journal of Fish Biology, 56(5): 1099-1114.

FLOETER, S. R., GUIMARÃES, R. Z., ROCHA, L. A., FERREIRA, C. E. L., RANGEL, C. A., \& GASPARINI, J. L. 2001. Geographic variation in reef-fish assemblages along the Brazilian coast. Global Ecology and Biogeography, 10(4): 423-431.
GOTANDA, K. M., TURGEON, K., \& KRAMER, D. L. 2009. Body size and reserve protection affect flight initiation distance in parrotfishes. Behavioral Ecology and Sociobiology, 63(11), 1563-1572.

HARVEY, E. S., FLETCHER, D. \& SHORTIS, M. 2002 Estimation of reef fish length by divers and by stereo-video: a first comparison of the accuracy and precision in the field on living fish under operational conditions. Fisheries Research 57: 255-265.

IBAMA, 1990. Plano de Manejo do Parque Nacional Marinho de Fernando de Noronha

ICMBio. Plano de Manejo APA Fernando de Noronha - Rocas - São Pedro São Paulo. http://www.icmbio.gov.br/portal/images/stories/servicos/A-_PM_APA_ Fernando_de_Noronha_-_Encartes_1_e_2.pdf. Acessado em 03.11.2019

ICMBio, 2018. Livro vermelho da fauna brasileira ameaçada de extinção. http:// icmbio.gov.br/portal/images/stories/comunicacao/publicacoes/publicacoesgdiversas/livro_vermelho_2018_vol1.pdf. Acessado em 04.11.2019

ILARRI, M. I., SOUZA, A. T., \& ROSA, R. S. 2017. Community structure of reef fishes in shallow waters of the Fernando de Noronha archipelago: effects of different levels of environmental protection. Marine and Freshwater Research, 68(7): 1303-1316.

IUCN, 2019. https://www.iucnredlist.org/. Acessado em 31.10.2019

JANUCHOWSKI-Hartley, F. A., GRAHAM, N. A., FEARY, D. A., MOROVE, T., \& CINNER, J. E. 2011. Fear of fishers: human predation explains behavioral changes in coral reef fishes. PLoS One, 6(8), e22761.

KRAJEWSKI, J. P., \& FLOETER, S. R. 2011. Reef fish community structure of the Fernando de Noronha Archipelago (Equatorial Western Atlantic): the influence of exposure and benthic composition. Environmental Biology of Fishes, 92(1): 25.

KULBICKI, M. 1998. How the acquired behaviour of commercial reef fishes may influence the results obtained from visual censuses. Journal of Experimental Marine Biology and Ecology 222: 11-30.

LINDFIELD, S. J., HARVEY, E. S., MCILWAIN, J. L. \& HALFORD, A. R. 2014. Silent fish surveys: bubble-free diving highlights inaccuracies associated with SCUBA-based surveys in heavily fished areas. Methods in Ecology and Evolution 5: 1061-1069.

MAIDA, M., FERREIRA, B.P. 1997. Coral reefs of Brazil: an overview. Proc. 8th Int. Coral Reef Symp.1997; 1: 263-274.

MEDEIROS, P. R., ROSA, R. S., \& FRANCINI-FILHO, R. B. 2011. Dynamics of fish assemblages on a continuous rocky reef and adjacent unconsolidated habitats at Fernando de Noronha Archipelago, tropical western Atlantic. Neotropical Ichthyology 9: 869-879.

MUSICK, J.A., BURGESS, G., CAILLIET, G., CAMHI, M. \& FORDHAM, S. 2000. Management of sharks and their relatives (Elasmobranchii). Fisheries 25: 9-13.

NUNES, J. D. A. C. D., MEDEIROS, D. V., REIS-FILHO, J. A., SAMPAIO, C. L. S. \& BARROS, F. 2012. Reef fishes captured by recreational spearfishing on reefs of Bahia State, northeast Brazil. Biota Neotropica 12: 179-185.

NUNES, J. D. A. C., LOIOLA, M., MIRANDA, R. J., SAMPAIO, C. L., \& BARROS, F. 2016. Are Abrolhos no-take area sites of naïve fish? An evaluation using flight initiation distance of labrids. Neotropical Ichthyology, 14(4).

PINHEIRO, H. T., ROCHA, L. A., MACIEIRA, R. M., CARVALHO-FILHO, A., ANDERSON, A. B., BENDER, M. G., ... \& GASPARINI, J. L. 2018. South-western Atlantic reef fishes: Zoogeographical patterns and ecological drivers reveal a secondary biodiversity centre in the Atlantic Ocean. Diversity and Distributions, 24(7): 951-965.

SOTO, J. M. R. 2001. Peixes do arquipélago Fernando de Noronha. Mare Magnum, 1(2): 147-169.

WILLIS, T. J., \& BABCOCK, R. C. 2000. A baited underwater video system for the determination of relative density of carnivorous reef fish. Marine and Freshwater Research 51(8): 755-763.

Received: 08/04/2020

Revised: 29/09/2020

Accepted: 06/10/2020

Published online: 21/12/2020 